Annals of Pure and Applied Mathematics

Vol. 14, No. 2, 2017, 347-352

ISSN: 2279-087X (P), 2279-0888(online)

Published on 30 September 2017

www.researchmathsci.org

DOI: http://dx.doi.org/10.22457/apam.v14n2a18

Annals of

Pure and Applied

Mathematics

\title{
Extremal Solution for Fractional Quadratic Integral Equation in Banach Space
}

\author{
B.D.Karande ${ }^{1}$ and S.S.Yachawad \\ ${ }^{1}$ Department of Mathematics, Maharashtra Udayagiri Mahavidyala, Udgir-413517 \\ Maharashtra, India. \\ Email: bdkarande@rediffmail.com \\ ${ }^{2} 102$, 'A' Wing, Raval Tower, Sector - 11, C.B.D. Belapur, Navi Mumbai- 400614 \\ Maharashtra, India. \\ Corresponding author. Email: sshelke1234@gmail.com
}

Received 14 September 2017; accepted 28 September 2017

Abstract. In this paper, we proved existence the extremal solution for a fractional order quadratic integral equation in Banach space under lipschitz and Caratheodory conditions via a hybrid fixed point theorem.

Keywords: Banach space, fractional order quadratic integral equation, existence results, fixed point theorem.

AMS Mathematics Subject Classification (2010): 47H30, 26A33, 46BXX

\section{Introduction}

The theory of fractional calculus has newly received a lot of attention and establishes a meaningful branch of nonlinear analysis. Number of research papers and research monographs has appeared devoted to integrals and differential equation of fractional order.

In this paper we study the existence of locally attractive solution of the following fractional order quadratic integral equation.

$x(t)=g(t, x(t))+\frac{f(t, x(t), x(\gamma(t)))}{\Gamma(\xi)} \int_{0}^{t} \frac{v(t, s, x(s))}{(t-s)^{1-\xi}} d s$

where $t \in \mathbb{R}_{+}=[0, \infty)$ and $0<\xi<1, g: \mathbb{R}_{+} \times \mathbb{R} \rightarrow \mathbb{R}, f: \mathbb{R}_{+} \times \mathbb{R} \times \mathbb{R} \rightarrow \mathbb{R}$ and $v: \mathbb{R}_{+} \times \mathbb{R}_{+} \times \mathbb{R} \rightarrow \mathbb{R}$ are functions which satisfy special assumptions.

\section{Preliminaries}

In this section, we introduce some notations and definitions of fractional calculus and present preliminary results needed in our proofs later.

Let $\mathbb{X}=\mathcal{A C}\left(\mathbb{R}_{+}, \mathbb{R}\right)$ be the space of absolutely continuous function on $\mathbb{R}_{+}$and $\Omega$ be a subset of $\mathbb{X}$. Let a mapping $\mathbb{A}: \mathbb{X} \rightarrow \mathbb{X}$ be an operator and consider the following operator equation in $\mathbb{X}$ namely,

$$
x(t)=(\mathbb{A} x)(t), \text { for all } t \in \mathbb{R}_{+}
$$




\section{B.D.Karande and S.S.Yachawad}

Below we give some different characterization of the solutions for operator equation (2) on $\mathbb{R}_{+}$. We need the following definitions.

Definition 2.1[3]. Let $\mathbb{X}$ be a Banach space. A mapping $\mathbb{A}: \mathbb{X} \rightarrow \mathbb{X}$ is called Lipschitz if there is a constant $\alpha>0$ such that, $\|\mathbb{A} x-\mathbb{A} y\| \leq \alpha\|x-y\|$ for all $x, y \in \mathbb{X}$. If $\alpha<1$, then $\mathbb{A}$ is called a contraction on $\mathbb{X}$ with the contraction constant $\alpha$.

Definition 2.2. $[2,3]$ An operator $\mathbb{Q}$ on a Banach space $\mathbb{X}$ into itself is called compact if for any bounded subset $S$ of $\mathbb{X}, \mathbb{Q}(S)$ is relatively compact subset of $\mathbb{X}$. If $\mathbb{Q}$ is continuous and compact, then it is called completely continuous on $\mathbb{X}$.

Definition 2.3. [4] Let $\mathbb{X}$ be a Banach space with the norm $\|\cdot\|$ and let $\mathbb{Q}: \mathbb{X} \rightarrow \mathbb{X}$, be an operator (in general nonlinear). Then $\mathbb{Q}$ is called

i. $\quad$ Compact if $\mathbb{Q}(X)$ is relatively compact subset of $\mathbb{X}$.

ii. $\quad$ Totally compact if $\mathbb{Q}(S)$ is totally bounded subset of $\mathbb{X}$ for any bounded subset $S$ of $\mathbb{X}$.

iii. Completely continuous if it is continuous and totally bounded operator on $\mathbb{X}$. It is clear that every compact operator is totally bounded but the converse need not be true.

We seek the solution of (2.1) in the space $\mathcal{A C}\left(\mathbb{R}_{+}, \mathbb{R}\right)$ of continuous and real - valued function defined on $\mathbb{R}_{+}$. Define a standard norm $\|\cdot\|$ and a multiplication "." in $\mathcal{A C}\left(\mathbb{R}_{+}, \mathbb{R}\right)$ by

$$
\|x\|=\sup \left\{|x(t)|: t \in \mathbb{R}_{+}\right\}, \quad(x y)(t)=x(t) y(t), \quad t \in \mathbb{R}_{+}
$$

Clearly $\mathcal{A C}\left(\mathbb{R}_{+}, \mathbb{R}\right)$ becomes a Banach space with respect to the above norm and the multiplication in it. By $\mathcal{L}^{1}\left(\mathbb{R}_{+}, \mathbb{R}\right)$ we denote the space of Lebesgue-integrable function $\mathbb{R}_{+}$with the norm $\|\cdot\|_{\mathcal{L}^{1}}$ defined by

$$
\|x\|_{\mathcal{L}}=\int_{0}^{\infty}|x(t)| d t
$$

Definition 2.4 [1]: Let $f \in \mathcal{L}^{1}[0, \mathbb{T}]$ and $\xi>0$. The Riemann-Liouville fractional derivative of order $\xi$ of real function $f$ is defined as

$$
\mathfrak{D}^{\xi} f(t)=\frac{1}{\Gamma(1-\xi)} \frac{d}{d t} \int_{0}^{t} \frac{f(s)}{(t-s)^{\xi}} d s \quad, \quad 0<\xi<1
$$

Such that $\mathfrak{D}^{-\xi} f(t)=I^{\xi} f(t)=\frac{1}{\Gamma(\xi)} \int_{0}^{t} \frac{f(s)}{(t-s)^{1-\xi}} d s$ respectively.

Definition 2.5. [1] The Riemann-Liouville fractional integral of order $\xi \in(0,1)$ of the function $f \in \mathcal{L}^{1}[0, \mathbb{T}]$ is defined by the formula: $I^{\xi} f(t)=\frac{1}{\Gamma(\xi)} \int_{0}^{t} \frac{f(s)}{(t-s)^{1-\xi}} d s, \quad t \in$ $[0, \mathbb{T}]$ where $\Gamma(\xi)$ denote the Euler gamma function. The Riemann-Liouville fractional derivative operator of order $\xi$ defined by $\mathfrak{D}^{\xi}=\frac{d^{\xi}}{d t^{\xi}}=\frac{d}{d t}{ }^{\circ} I^{1-\xi}$. It may be shown that the fractional integral operator $I^{\xi}$ transforms the space $\mathcal{L}^{1}\left(\mathbb{R}_{+}, \mathbb{R}\right)$ into itself and has some other properties. 
Extremal Solution for Fractional Quadratic Integral Equation in Banach Space

Theorem 2.1. [4] (Arzela-Ascoli Theorem) If every uniformly bounded and equi continuous sequence $\left\{f_{n}\right\}$ of functions in $\mathcal{C}\left(\mathbb{R}_{+}, \mathbb{R}\right)$, then it has a convergent subsequence.

Theorem 2.2. [4] A metric space $X$ is compact iff every sequence in $X$ has a convergent subsequence.

Theorem 2.3. (Lebesgue's dominated convergence theorem) Suppose that $\left\{g_{n}\right\}$ is a sequence of measurable functions, that $g_{n} \rightarrow g$ pointwise a.e. as $n \rightarrow \infty$, and that $\left|g_{n}\right| \leq f, \forall n$, where $f$ is integrable then $g$ is integrable and $\int g d \mu=\lim _{n \rightarrow \infty} \int g_{n} d \mu$.

Definition 2.6. [6] A closed and non-empty set $\mathbb{K}$ in a Banach Algebra $\mathbb{X}$ is called a cone if,

i. $\quad \mathbb{K}+\mathbb{K} \subseteq \mathbb{K}$

ii. $\lambda \mathbb{K} \subseteq \mathbb{K}$ for $\lambda \in \mathbb{R}, \lambda \geq 0$

iii. $\quad\{-\mathbb{K}\} \cap \mathbb{K}=0$ where 0 is the zero element of $\mathbb{X}$.

and is called positive cone if

iv. $\quad \mathbb{K} \circ \mathbb{K} \subseteq \mathbb{K}$

and the notation $\circ$ is a multiplication composition in $\mathbb{X}$. We introduce an order relation $\leq$ in $\mathbb{X}$ as follows.

Let $x, y \in \mathbb{X}$ then $x \leq y$ if and only if $y-x \in \mathbb{K}$. A cone $\mathbb{K}$ is called normal if the norm $\|\cdot\|$ is monotone increasing on $\mathbb{K}$. It is known that if the cone $\mathbb{K}$ is normal in $\mathbb{X}$ then every order-bounded set in $\mathbb{X}$ is norm-bounded set in $\mathbb{X}$. The details of cone and their properties appear in Guo and Lakshikantham [7].

We equip the space $\mathcal{C}(\mathbb{J}, \mathbb{R})$ of continuous real valued function on $\mathbb{R}_{+}$with the order relation $\leq$ with the help of cone defined by,

$\mathbb{K}=\{x \in \mathcal{C}(\mathbb{J}, \mathbb{R}): x(t) \geq 0 \forall t \in \mathbb{J}\}$

We well known that the cone $\mathbb{K}$ is normal and positive in $\mathcal{C}(\mathbb{J}, \mathbb{R})$. As a result of positivity of the cone $\mathbb{K}$ we have:

Lemma 2.1. [8] Let $p_{1}, p_{2}, q_{1}, q_{2} \in \mathbb{K}$ be such that $p_{1} \leq q_{1}$ and $p_{2} \leq q_{2}$ then $p_{1} p_{2} \leq q_{1} q_{2}$. given by,

For any $p, q \in \mathbb{X}=\mathcal{C}\left(\mathbb{R}_{+}, \mathbb{R}\right), p \leq q$ the order interval $[p, q]$ is a set in $\mathbb{X}$

$$
[p, q]=\{x \in \mathbb{X}: p \leq x \leq q\}
$$

Definition 2.7. [6] A mapping $G:[p, q] \rightarrow \mathbb{X}$ is said to be nondecreasing or monotone increasing if $x \leq y$ implies $G x \leq G y$ for all $x, y \in[p, q]$.

We use following fixed point theorem of Dhage [8] for proving the existence of extremal solution for the FQIE (1.1) under certain monotonicity conditions.

Theorem 2.4. [8] Let $\mathbb{K}$ be a cone in Banach Algebra $\mathbb{X}$ and let $[p, q] \in \mathbb{X}$. Suppose that $\mathbb{A}, \mathbb{B}:[p, q] \rightarrow \mathbb{K}$ and $\mathbb{C}:[p, q] \rightarrow \mathbb{X}$ be three nondecreasing operators such that

a. $\mathbb{A}$ and $\mathbb{C}$ are a Lipschitz with Lipschitz constant $\alpha, \beta$

b. $\mathbb{B}$ is completely continuous,

c. The elements $p, q \in \mathbb{X}$ satisfy $p \leq \mathbb{A} p \mathbb{B} p+\mathbb{C} p$ and $\mathbb{A} q \mathbb{B} q+\mathbb{C} q \leq q$ 


\section{B.D.Karande and S.S.Yachawad}

Further if the cone $\mathbb{K}$ is normal and positive then the operator equation $x=\mathbb{A} x \mathbb{B} y+$ $\mathbb{C} x$ has the least and greatest positive solution in $[p, q]$ whenever $\alpha M+\beta<1$, where $M=\|\mathbb{B}([p, q])\|=\sup \{\|\mathbb{B} x\|: x \in[p, q]\}$.

\section{Existence theory}

Let $\mathbb{X}=\mathcal{C}\left(\mathbb{R}_{+}, \mathbb{R}\right)$ be a space of continuous real valued functions defined on $\mathbb{R}_{+}$. Define a norm $\|\cdot\|$ and a multiplication in $\mathbb{X}$ by, $\|x\|=\sup _{t \in \mathbb{R}_{+}}|x(t)|$ and $(x y)(t)=$ $(t) \mathcal{y}(t), \forall t \in \mathbb{R}_{+}$.

Clearly $\mathbb{X}$ is Banach Algebra with respect to above supremum norm and multiplication in it.

Definition 3.1. [9] A mapping $v: \mathbb{R}_{+} \times \mathbb{R}_{+} \times \mathbb{R} \rightarrow \mathbb{R}$ is Caratheodory if:

i) $\quad(t, s) \rightarrow v(t, s, x)$ is measurable for each $x \in \mathbb{R}$ and

ii) $\quad(x) \rightarrow v(t, s, x)$ is continuous almost everywhere for $t \in \mathbb{R}_{+}$.

Furthermore a Caratheodary function $v$ is $\mathcal{L}^{1}$-Caratheodary if:

iii) For each real number $r>0$ there exists a function $h_{r} \in \mathcal{L}^{1}\left(\mathbb{R}_{+} \times \mathbb{R}_{+}, \mathbb{R}\right)$ such that $|v(t, s, x)| \leq h_{r}(t, s)$ a.e. $t \in \mathbb{R}_{+}$for all $x \in \mathbb{R}$ with $|x|_{r} \leq r$.

Finally a caratheodary function $v$ is $\mathcal{L}_{\mathbb{X}}^{1}$-caratheodary if:

iv) There exists a function $h \in \mathcal{L}^{1}\left(\mathbb{R}_{+} \times \mathbb{R}_{+}, \mathbb{R}\right)$ such that $|v(t, s, x)| \leq$ $h(t, s)$, a.e. $t \in \mathbb{R}_{+}$for all $x \in \mathbb{R}$

For convenience, the function $h$ is referred to as a bound function for $v$.

\section{Existence of extremal solutions}

We need following definitions in sequel.

Definition 4.1. A function $p \in \mathcal{A C}\left(\mathbb{R}_{+}, \mathbb{R}\right)$ is called a lower solution of the FQIE (1) on $\mathbb{R}_{+}$if the function $t \rightarrow\left\{\frac{p(t)-g(t, p(t))}{f(t, p(t), p(\gamma(t)))}\right\}$ is continuous absolutely and

$$
p(t) \leq g(t, p(t))+\frac{f(t, p(t), p(\gamma(t)))}{\Gamma(\xi)} \int_{0}^{t} \frac{v(t, s, p(s))}{(t-s)^{1-\xi}} d s
$$

Again a function $q \in \mathcal{A C}\left(\mathbb{R}_{+}, \mathbb{R}\right)$ is called an upper solution of the FQIE (1) on $\mathbb{R}_{+}$if the function $t \rightarrow\left\{\frac{q(t)-g(t, q(t))}{f(t, q(t), q(\gamma(t)))}\right\}$ is continuous absolutely and

$q(t) \geq g(t, q(t))+\frac{f(t, q(t), q(\gamma(t)))}{\Gamma(\xi)} \int_{0}^{t} \frac{v(t, s, q(s))}{(t-s)^{1-\xi}} d s$

Definition 4.2. A solution $x_{M}$ of the FQIE (1) is said to be maximal if for any other solution $x$ to FQIE (1) has $x(t) \leq x_{M}(t)$ for all $t \in \mathbb{R}_{+}$. Again a solution $x_{M}$ of the FQIE (1) is said to be minimal if $x_{M}(t) \leq x(t)$ for all $t \in \mathbb{R}_{+}$where $x$ is any solution of the FQIE (1) on $\mathbb{R}_{+}$.

Definition 4.3. (Caratheodory case) A function $\sigma: \mathbb{R} \rightarrow \mathbb{R}$ is nondecreasing if $\sigma(x) \leq$ $\sigma(y) \forall x, y \in \mathbb{R}$ for which $x \leq y$. Similarly $\sigma(x)$ is increasing in $x$ if $\sigma(x)<$ $\sigma(y) \forall x, y \in \mathbb{R}$ for which $x<y$.

We consider the following assumptions:

$\mathfrak{B}_{\mathbf{1}}$ ) The function $x \rightarrow\left\{\frac{x(t)-g(t, x(t))}{f(t, x(t), x(\gamma(t)))}\right\}$ is increasing in the interval 
Extremal Solution for Fractional Quadratic Integral Equation in Banach Space

$$
\left[\min _{t \in \mathbb{R}_{+}} p(t), \max _{t \in \mathbb{R}_{+}} q(t)\right] \text {. }
$$

$\mathfrak{B}_{2}$ ) The functions $g: \mathbb{R}_{+} \times \mathbb{R} \rightarrow \mathbb{R}, f: \mathbb{R}_{+} \times \mathbb{R} \times \mathbb{R} \rightarrow \mathbb{R}$ and $v: \mathbb{R}_{+} \times \mathbb{R}_{+} \times \mathbb{R} \rightarrow \mathbb{R}$ are on decreasing in $x$ almost everywhere for $t \in \mathbb{R}_{+}$.

$\mathfrak{B}_{3}$ ) The FQIE (1) has a lower solution $p$ and upper solution $q$ on $\mathbb{R}_{+}$with $p \leq q$.

$\mathfrak{B}_{4}$ ) The function $v(t, s, x(s))$ is caratheodory.

$\left.\mathfrak{B}_{5}\right)$ The function $l: \mathbb{R}_{+} \rightarrow \mathbb{R}$ defined by $l(t)=|v(t, s, p(s))|+|v(t, s, q(s))|$ is Lebesgue measurable.

Remark 4.1. Assume that the hypotheses $\left(\mathfrak{B}_{1}-\mathfrak{B}_{4}\right)$ holds, then the function $t \rightarrow$ $v(t, s, x(s))$ is lebesgue integrable on $\mathbb{J}$, say $|v(t, s, x(s))| \leq l(t)$, a.e., $t \in \mathbb{R}_{+}$, for all $x \in[p, q]$ and some lebesgue integrable function $l$.

Theorem 4.1. Assume that the hypothesis $\left(\mathfrak{B}_{1}-\mathfrak{B}_{4}\right)$ holds and $l$ is given in above remark, further $\|\alpha\| \frac{1}{\Gamma(\xi+1)} T^{\xi}\|l\|_{\mathcal{L}^{1}}+\|\beta\|<1$, then FQIE (1) has minimal and maximal positive solution on $\mathbb{R}_{+}$.

Proof: Let $\mathbb{X}=\mathcal{C}(\mathbb{J}, \mathbb{R})$ and we define an order relation " $\leq$ " by the cone $\mathbb{K}$ given by (5). Clearly $\mathbb{K}$ is a normal cone in $\mathbb{X}$.

Now we define three operators $\mathbb{A}, \mathbb{B}:[p, q] \rightarrow \mathbb{K}$ and $\mathbb{C}:[p, q] \rightarrow \mathbb{X}$ by,

$\mathbb{A} x(t)=f(t, x(t), x(\gamma(t))), t \in \mathbb{R}_{+}$

$\mathbb{B} x(t)=\frac{1}{\Gamma(\xi)} \int_{0}^{t} \frac{v(t, s, x(s))}{(t-s)^{1-\xi}} d s, t \in \mathbb{R}_{+}$

$\mathbb{C} x(t)=g(t, x(t)), t \in \mathbb{R}_{+}$

The FQIE is equivalent to the operator equation

$x(t)=\mathbb{A} x(t) \mathbb{B} x(t)+\mathbb{C} x(t), \forall t \in \mathbb{R}_{+}$

Theorem (2.4).

We shall show that, the operators $\mathbb{A}, \mathbb{B}$ and $\mathbb{C}$ satisfy all the conditions of

This will be achieved in the following series of steps.

Notice that $\left(\mathfrak{B}_{2}\right)$ implies $\mathbb{A}, \mathbb{B}:[p, q] \rightarrow \mathbb{K}$ also note that $\left(\mathfrak{B}_{3}\right)$ ensures that $p \leq$ $\mathbb{A} p \mathbb{B} p+\mathbb{C} p$ and $\mathbb{A} q \mathbb{B} q+\mathbb{C} q \leq q$. Since the cone $\mathbb{K}$ in $\mathbb{X}$ is normal, $[p, q]$ is a norm bounded set in $\mathbb{X}$. Now it is shown, as in the proof of theorem (4.1) in the paper [5], that $\mathbb{A}$ and $\mathbb{C}$ are Lipschitz with a Lipschitz constant \|\|$\zeta\|\| \|$ and \|\|$\eta\|\| \|$ respectively. Similarly $\mathbb{B}$ is completely continuous operator on $[\not p, q]$. Again the hypothesis $(\mathfrak{B} 2)$ implies that $\mathbb{A}, \mathbb{B}$ and $\mathbb{C}$ are non-decreasing on $[p, q]$.

Step I: To show that the operators $\mathbb{A}, \mathbb{B}$ and $\mathbb{C}$ are non-decreasing on $[p, q]$. let $x, y \in$ $[p, q]$ be such that $x \leq y$.

$$
\begin{aligned}
& \mathbb{A} x(t)=f(t, x(t), x(\gamma(t))) \leq f(t, y(t), y(\gamma(t))) \leq \mathbb{A} y(t), \forall t \in \mathbb{R}_{+} \\
& \mathbb{B} x(t)=\frac{1}{\Gamma(\xi)} \int_{0}^{t} \frac{v(t, s, x(s))}{(t-s)^{1-\xi}} d s \leq \frac{1}{\Gamma(\xi)} \int_{0}^{t} \frac{v(t, s, y(s))}{(t-s)^{1-\xi}} d s \leq \mathbb{B} y(t), t \in \mathbb{R}_{+}
\end{aligned}
$$

Step II: Again definition (4.1) and hypothesis $\left(\mathfrak{B}_{3}\right)$ implies that

$$
p(t) \leq g(t, p(t))+\frac{f(t, p(t), p(\gamma(t)))}{\Gamma(\xi)} \int_{0}^{t} \frac{v(t, s, p(s))}{(t-s)^{1-\xi}} d s
$$


B.D.Karande and S.S.Yachawad

and $x \in[p, q]$

$$
\begin{aligned}
& \leq g(t, x(t))+\frac{f(t, x(t), x(\gamma(t)))}{\Gamma(\xi)} \int_{0}^{t} \frac{v(t, s, x(s))}{(t-s)^{1-\xi}} d s \\
& \leq g(t, q(t))+\frac{f(t, q(t), q(\gamma(t)))}{\Gamma(\xi)} \int_{0}^{t v\left(t, s, q, q_{(}\right)} \frac{1}{(t-s)^{1-\xi}} d s \leq q(t), \forall t \in \mathbb{R}_{+}
\end{aligned}
$$

As a result $p(t) \leq \mathbb{A} x(t) \mathbb{B} x(t)+\mathbb{C} x(t) \leq q(t), \forall t \in \mathbb{R}_{+}$and $x \in[p, q]$

Hence $\mathbb{A} x \mathbb{B} x+\mathbb{C} x \in[p, q], \forall x \in[p, q]$

Step III: Again $M=\|\mathbb{B}([p, q])\|=\sup \{\|\mathbb{B} x\|: x \in[p, q]\}$

$$
\begin{aligned}
& \leq \sup \left\{\sup _{t \in \mathbb{R}_{+}} \int_{0}^{t} \frac{1}{\Gamma(\xi)}(t-s)^{\xi-1}|v(t, s, x(s))|: x \in[p, q]\right\} \\
& \leq \sup \frac{1}{\Gamma(\xi)}\left[\frac{(t-s)^{\xi}}{\xi}\right]_{0}^{t}\|l\|_{\mathcal{L}^{1}} \leq \frac{1}{\Gamma(\xi+1)} T^{\xi}\|l\|_{\mathcal{L}^{1}}
\end{aligned}
$$

Since $\alpha M+\beta<\|\alpha\| \frac{1}{\Gamma(\xi+1)} T^{\xi}\|l\|_{\mathcal{L}^{1}}+\|\beta\|<1$

We apply Theorem 2.4 to the operator equation $\mathbb{A} x \mathbb{B} x+\mathbb{C} x=x$ to yield that the FQIE (1.1) has minimum and maximum positive solution on $\mathbb{R}_{+}$. This completes the proof.

\section{Conclusion}

In this paper, we have studied the existence the extremal solution for fractional quadratic integral equation. The result has been obtained by using hybrid fixed point theorem for three operators in Banach space due to Dhage.

\section{REFERENCES}

1. S.Samko, A.A.Kilbas and O.Marivchev, Fractional Integrals and Derivative: Theory and Applications, Gordon and Breach, Amsterdam (1993).

2. J.Dugundji and A.Granas, Fixed Point Theory, in. Monographie Math., Warsaw (1982).

3. M.I.Abbas, On the Existence of locally attractive solution of a nonlinear quadratic voltera integral equation of fractional order, Advances in Difference Equations, 2010 (2010) 1-11.

4. B.D.Karande, Fractional order functional integro-differential equation in Banach Algebras, Malaysian Journal of Mathematical Sciences, 8 (2013) 1-16.

5. B.D.Karande and S.S.Yachawad, Attractivity result for fractional quadratic integral equation in banach space, International Journal of Mathematical Archive, 8(6) (2017) 125-132.

6. B.C.Dhage, Nonlinear functional boundary value problems in Banach Algebras involving Caratheodories, Kyungpook Math. J., 46 (2006) 527-541.

7. D.Guo and V.Lakshmikantham, Nonlinear problems in abstract spaces, Academic Press, (1982).

8. B.C.Dhage, Periodic boundary value problems of first order Caratheodory and discontinuous differential equation, Nonlinear Func. Anal. Appl., 13(2) (2008) 323352.

9. J.Banas and B.Rzepka, Monotonic solution of a quadratic integral equation of fractional order, J. Math. Anal. Appl., 332 (2007) 1371-1379. 